\title{
Judging interevent relations: From cause to effect and from effect to cause
}

\author{
LINDA J. VAN HAMME, SHU-FANG KAO, and EDWARD A. WASSERMAN \\ University of Iowa, lowa City, Iowa
}

\begin{abstract}
Stimulus competition was studied in college students' correlational judgments in a medical decision-making setting. In accord with prior findings, subjects making cause-to-effect (predictive) judgments discounted a stimulus event that was moderately correlated with a target event when rival stimuli were more highly correlated with the effect. However, subjects making effectto-cause (diagnostic) judgments were not at all disposed to discount a stimulus event which was moderately correlated with a target event when rival stimuli were more highly correlated with the cause. The theoretical implications of these results are considered in connection with associative and mentalistic models of causal attribution.
\end{abstract}

In the past several years, there has been a growing interest in assessing the utility of elementary associative principles to elucidate such advanced forms of human cognition as causal inference and categorization (Chapman \& Robbins, 1990; Gluck \& Bower, 1988; Shanks \& Dickinson, 1987; Wasserman, 1990b). This general strategy runs counter to much work in contemporary cognitive psychology, but it is quite consistent with the emerging school of connectionism (Rumelhart \& McClelland, 1986). Although some may resist the possibility that common psychological processes participate in the behavior of both human and nonhuman animals, the real questions are whether complex cognitive processes build upon or derive from simpler ones, such as interevent association.

Before answering these questions, it seems proper to assess the generality of many basic associative principles in both human and nonhuman behavior. One such principle-associative competition-is that the relative as well as the absolute predictiveness of a stimulus affects its association with another event. So, a stimulus, $X$, which is followed by an effect $50 \%$ of the time may be differentially associated with the effect, depending on the relative predictiveness of other stimuli, $A$ and $B$, which are presented in compound with $X$. If $A X$ compounds are always followed by the effect $(A X-100 \%)$ and $B X$ compounds are never followed by the effect $(B X-0 \%)$, then $X$ is more weakly associated with the effect than when $A X$ and $B X$ compounds are equally often followed by the effect $(A X$ $50 \%$ and $B X-50 \%$ ). Critically, the absolute predictiveness of the effect by $X$ is exactly the same in both conditions, thereby implicating the relative predictiveness of $A$ and $B$ in the association of $X$ with the effect.

This competitive association principle was originally documented by Wagner and his associates in Pavlovian and operant conditioning experiments with rats and rabbits

Correspondence concerning this article should be addressed to Edward A. Wasserman, University of Iowa, Iowa City, IA 52242.
(Wagner, Logan, Haberlandt, \& Price, 1968). Wasserman later extended these findings to autoshaping procedures with pigeons (Wasserman, 1974). But most relevant to the present discussion is the fact that precisely the same form of stimulus competition was observed when college students were asked to rate the causal connection between a hypothetical patient's allergic reaction and the patient's prior consumption of three different foods compounded in meals of the form $A X$ and $B X$ (Wasserman, 1990a).

This empirical convergence in the behavior of humans and nonhumans may indicate the operation of common cognitive processes in associative learning and causal attribution (Shanks, 1991b), a possibility which would have heartened David Hume, who originally proposed an associative account of causal perception (Fales \& Wasserman, 1992). But, just how general is this competition in the causal attribution process? Specifically, is competition as readily observed when subjects reason from multiple effects to single causes as when they reason from multiple causes to single effects?

The question of competitive symmetry arises because of recent research by Waldmann and Holyoak (1990, 1992), which suggests that cue competition effects-an important feature of current associative learning theories such as that of Rescorla and Wagner (1972)-occur only when subjects reason from multiple possible causes to a single effect (causes compete in predictive judgments), but not when subjects reason from multiple effects to a single possible cause (effects do not compete in diagnostic judgments). Waldmann and Holyoak argue that diagnostic causal judgments represent a more abstract form of inference which cannot be explained by associative mechanisms. However, it is not clear to us why the ability of associative models to explain stimulus selection effects would justify the conclusion that associative processes cannot be involved in the (actually simpler) learning of noncompetitive stimulus-outcome relations. Earlier contiguity theories of associative learning (Hull, 1943; Spence, 1956) or applications of the Rescorla-Wagner model to situations 
in which there is only a single conditioned stimulus (CS) could easily accommodate these noncompetitive effects.

The Rescorla-Wagner associative model was designed to explain the stimulus selection effects that occur when multiple CSs are paired with one unconditioned stimulus (US). In classical conditioning, the CS precedes the US (as a cause precedes an effect), and multiple simultaneously presented CSs will compete for associative strength with the US (i.e., they will compete to predict the US). The Rescorla-Wagner model does not imply the inverse relation-that multiple USs will compete for association with one CS. A single CS should be able to acquire full associative strength with any number of USs. This mathematical and logical property of the Rescorla-Wagner model has also been supported empirically by Rescorla (1991) with rats. Competitive asymmetry between causes and effects is therefore consistent with an associative account and would not appear to require the elaborate cognitive explanations of Waldmann and Holyoak.

The present experiment sought further to test whether competitive asymmetry is observed between problemsolving situations in which subjects reason from effect to cause and situations in which subjects reason from cause to effect. Shanks (1991a, Experiment 3) recently reported the results of an experiment in which stimulus competition was observed in a situation in which subjects reasoned from effects to causes. Since that experiment seems to contradict Waldmann and Holyoak's results regarding the asymmetrical nature of cue competition between causes and effects, further investigation of that asymmetry appears to be necessary.

To replicate our own prior work in human causal attribution (Wasserman, 1990a), half of the present subjects were given the task of determining the source of a hypothetical patient's allergic reaction after eating different combinations of foods. College students were asked to judge the efficacy of three foods in causing a patient's allergic reaction under a variety of different experimental arrangements. Five different conditions varied the differential correlation of $A X$ and $B X$ combinations with the occurrence and nonoccurrence of the patient's allergic reaction. At one extreme, the $A X$ and $B X$ combinations were equally (50\%) paired with the occurrence and nonoccurrence of the allergic reaction; thus, the difference in the probability of the allergic reaction after the two stimulus combinations was .00 . At the other extreme, the $A X$ compound was always $(100 \%)$ paired with the occurrence of the allergic reaction and the $B X$ compound was never $(0 \%)$ paired with the occurrence of the allergic reaction; thus, the difference in the probability of the allergic reaction after the two stimulus combinations was 1.00 . The three remaining conditions entailed intermediate $A X-B X$ correlations of $.25, .50$, and .75 , brought about by different pairings of $A X$ and $B X$ with the occurrence of the allergic reaction: $62.5 \%-37.5 \%, 75 \%-25 \%$, and $87.5 \%-12.5 \%$, respectively.

The other half of the subjects were given a different task. They were first told whether or not a hypothetical patient had eaten a particular food at meal time; then they were told what symptoms had followed meals containing that food and meals missing that food. The three symptoms occurred in the form of $A X$ and $B X$ compounds. The subjects were asked to rate the extent to which each particular symptom was a characteristic effect of eating the specific food. Five experimental conditions were again created in which now the $A X$ and $B X$ compounds were differentially correlated with the eating of a particular food. Here, however, multiple effects were differentially correlated with a single cause, whereas in the earlier case multiple causes were differentially correlated with a single effect.

Of interest was whether subjects in each group would sensitively rate the absolute correlation of the $A$ and $B$ elements with the target event (the effect for the first set of subjects and the cause for the second set of subjects). Of even greater interest was whether subjects in each group would differently rate the $X$ element depending on the differential correlation of the $A$ and $B$ elements with the target event.

\section{METHOD}

\section{Subjects}

The subjects were 950 students enrolled in introductory psychology courses at The University of Iowa. The subjects participated in the study in group testing sessions to fulfill a course requirement. There were 401 men and 549 women in the study.

\section{Materials}

Ten different kinds of experimental sheets were prepared, corresponding with the unique combinations of five different levels of compound stimulus correlation $(.00, .25, .50, .75$, and 1.00$)$ and two different problem contexts (cause-effect, or CE, and effectcause, or EC). Each subject received one sheet.

Subjects in Group CE were given the following prefatory paragraph: "Imagine that you are an allergist who is trying to determine the cause of an allergic reaction shortly after your patient eats dinner. You arrange that the patient eat particular foods at dinner over a series of evenings, and then report to you whether an allergic reaction followed. The results of the test series are shown below:'

Subjects in Group EC were given the following prefatory paragraph: "Imagine that you are an allergist who is trying to determine the nature of the allergic reaction that shortly follows the eating of shrimp. Over a series of evenings, one of your patients recorded the symptoms that followed dinners which did or did not include a portion of shrimp. The results of the series of dinners are shown below:"

All subjects were then given a listing of the results displayed in 16 rows, 1 row for each meal. For subjects in Group CE, each row showed the occurrence or nonoccurrence of Food 1 (shrimp, element $X$ ), Food 2 (strawberries, element $A$ ), Food 3 (peanuts, element $B$ ), and the allergic reaction. For subjects in Group EC, each row showed the occurrence or nonoccurrence of shrimp consumption during the meal, Symptom 1 (headache, element $X$ ), Symptom 2 (fever, element $\boldsymbol{A}$ ), and Symptom 3 (rash, element $B$ ). Table 1 illustrates the specific lists given to subjects in each of the 10 subgroups of the experiment

Following the results of the test series, the subjects were asked on their problem sheets to rate the relations of the $A, B$, and $X$ stimuli with the target event. Subjects in Group CE were asked to indicate their determination of the allergic reaction by choosing the appropriate numbers along three rating scales: one for shrimp, one for strawberries, and one for peanuts. Each scale ranged from 0 to 8 , with the following verbal descriptions at selected points along the 
Table 1

Information Given to Subjects in Groups CE and EC Group

Cause-Effect/Effect-Cause Allergic Reaction?/Shrimp Eaten?

\begin{tabular}{rlllllll} 
Day & Foods & .00 & .25 & .50 & .75 & 1.00 & Symptoms \\
\hline 1 & $A X$ & Yes & Yes & Yes & Yes & Yes & $A X$ \\
2 & $B X$ & No & No & No & No & No & $B X$ \\
3 & $B X$ & Yes & Yes & Yes & No & No & $B X$ \\
4 & $A X$ & No & Yes & Yes & Yes & Yes & $A X$ \\
5 & $A X$ & No & No & No & No & Yes & $A X$ \\
6 & $B X$ & Yes & Yes & No & No & No & $B X$ \\
7 & $A X$ & Yes & Yes & Yes & Yes & Yes & $A X$ \\
8 & $B X$ & No & No & No & No & No & $B X$ \\
9 & $B X$ & Yes & Yes & Yes & Yes & No & $B X$ \\
10 & $A X$ & No & No & Yes & Yes & Yes & $A X$ \\
11 & $B X$ & No & No & No & No & No & $B X$ \\
12 & $A X$ & Yes & Yes & Yes & Yes & Yes & $A X$ \\
13 & $A X$ & No & No & No & Yes & Yes & $A X$ \\
14 & $B X$ & Yes & No & No & No & No & $B X$ \\
15 & $A X$ & Yes & Yes & Yes & Yes & Yes & $A X$ \\
16 & $B X$ & No & No & No & No & No & $B X$ \\
\hline
\end{tabular}

scale: (0) definitely not, (4) possibly, and (8) definitely the cause of the allergic reaction. Subjects in Group EC were asked to indicate their characterization of the allergic reaction by choosing the appropriate numbers along three rating scales: one for headache, one for fever, and one for rash. Each scale ranged from 0 to 8, with the following verbal descriptions at selected points along the scale: (0) definitely not, (4) possibly, and (8) definitely the effect of eating shrimp.

\section{Procedure}

After giving their informed consent to participate in the group testing session, subjects immediately began the present experimental procedure. Random distribution of test materials resulted in $\mathbf{4 7 2}$ subjects in Group CE and 478 subjects in Group EC. For each of the 10 combinations of compound correlation and problem context, no fewer than 90 nor more than 98 subjects served. It took from 3 to $6 \mathrm{~min}$ for the subjects to read the instructions, to evaluate the test materials, and to make the three ratings.

\section{RESULTS}

Before examining the data of greatest interest-ratings of element $X$-the ratings of elements $A$ and $B$ will be considered in order to see whether changes in the correlation of $A X$ and $B X$ with the target event affected subjects' judgments.

\section{Ratings of $A$ and $B$}

The top and bottom panels of Figure 1 show the mean ratings of elements $A$ and $B$ in Groups $C E$ and $E C$, respectively. In each case, ratings of element $A$ rose and ratings of element $B$ fell as the difference in the correlation of $A X$ and $B X$ with the target event increased. Separate correlation (5) $\times$ group (2) $\times$ sex (2) analyses of variance assessed the reliability of each trend.

The overall increase in ratings of element $A$ was statistically reliable $[F(4,930)=109.69, p<.001]$. In addition, ratings of element $A$ were higher overall in Group CE than in Group EC $[F(1,930)=12.93, p<.001]$. No other main effects or interactions were significant. Followup analyses were undertaken to assess the separate reliabilities of the $A X-B X$ correlation main effect in Groups $\mathrm{CE}$ and EC. Each main effect was statistically significant [in Group CE, $F(4,462)=59.27, p<.001$, and in Group EC, $F(4,468)=51.57, p<.001]$.
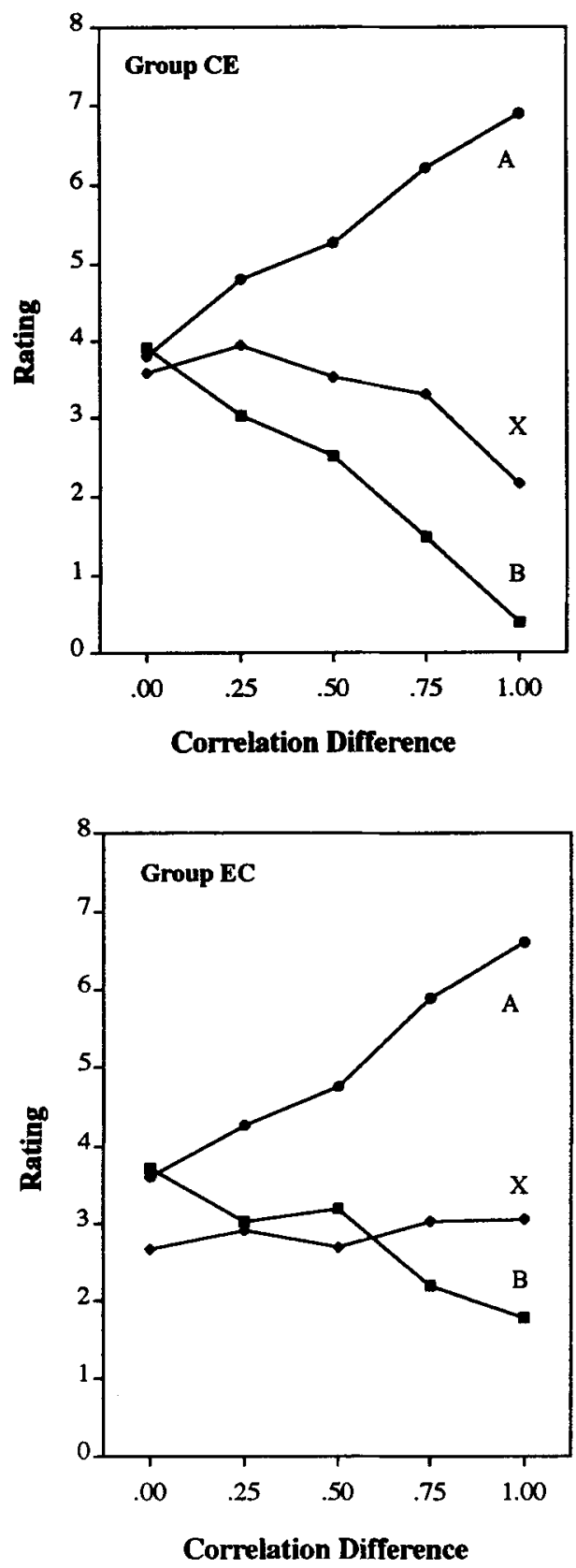

Figure 1. (Top) Mean causal rating scores of subjects in Group CE to elements $A, B$, and $X$ of $A X$ and $B X$ compounds as a function of the difference in the correlation of those compounds $(A X-B X)$ with the occurrence and nonoccurrence of an allergic reaction. (Bottom) Mean effect rating scores of subjects in Group EC to elements $A, B$, and $X$ of $A X$ and $B X$ compounds as a function of the difference in the correlation of those compounds $(A X-B X)$ with the consumption or nonconsumption of a food item. 
The overall decrease in ratings of element $B$ was statistically reliable $[F(4,930)=78.90, p<.001]$. In addition, ratings of element $B$ were lower overall in Group CE than in Group EC $[F(1,930)=21.60, p<$ $.001]$, and the interaction between correlation and group was significant $[F(4,930)=6.76, p<.001]$. No other main effects or interactions were significant. Follow-up analyses were undertaken to assess the separate reliabilities of the $A X-B X$ correlation main effect in Groups CE and EC. Each main effect was statistically significant [in Group CE, $F(4,462)=78.30, p<.001$, and in Group EC, $F(4,468)=18.10, p<.001]$.

Thus, ratings of elements $A$ and $B$ indicated that subjects were highly sensitive to differences in the correlation of $A X$ and $B X$ compounds with the occurrence and nonoccurrence of the target event under both methods of presenting the interevent information.

\section{Ratings of $\boldsymbol{X}$}

The top and bottom panels of Figure 1 also depict mean ratings of element $X$ in Groups $C E$ and EC, respectively. In Group CE, ratings of element $X$ fell as the $A X-B X$ correlation was increased (Figure 1, top); however, in Group EC, ratings of element $X$ were essentially unaffected by increases in the $A X-B X$ correlation (Figure 1, bottom).

Analysis of variance disclosed statistically reliable main effects of the $A X-B X$ correlation $[F(4,930)=3.30, p<$ $.05]$ and group $[F(1,930)=9.09, p<.01]$ and a reliable correlation by group interaction $[F(4,930)=5.69$, $p<.001]$. Follow-ups disclosed that Group CE evidenced a reliable main effect of the $A X-B X$ correlation on ratings of element $X[F(4,462)=10.38, p<.001]$ but that Group EC did not $[F(4,468)<1]$.

\section{DISCUSSION}

The results of subjects in Group CE closely match those of subjects in the study by Wasserman (1990a, Group Series). In different conditions of both projects, $A X$ and $B X$ causal compounds were differentially correlated with the occurrence of a hypothetical patient's allergic reaction. College students' ratings of the causal efficacy of the common $X$ element were higher when the $A X$ and $B X$ compounds were equally correlated with the occurrence and nonoccurrence of the allergic reaction than when $A X$ was always paired with the occurrence of the allergic reaction and $B X$ was never paired with the occurrence of the allergic reaction. Such differential responding to element $X$ held despite its equivalent pairing with the allergic reaction in each of the different correlation conditions. Once again, humans' causal judgments exhibited a selective attributional effect parallel to that evidenced by the rats, rabbits, and pigeons whose conditioned responses were measured by Wagner et al. (1968) and by Wasserman (1974). This parallel was not only qualitative, but quantitative; responding to element $X$ was inversely related to the difference in outcome probability after $A X$ and $B X$ compounds for the conditioned keypecks of pigeons and for the causal ratings of humans (see Figure 1 in Wasserman, 1990a, and Figure 1 here).

The results of subjects in Group EC were decidedly different. Here, responding to element $X$ of symptom compounds was completely unaffected by the differential correlation with the cause of elements $A$ and $B$ from $A X$ and $B X$ compounds (Figure 1). Such nondifferential responding to element $X$ cannot be accounted for by subjects' failure to appreciate the differential correlation of elements $A$ and $B$ with the cause; ratings of element $A$ appropriately rose and ratings of element $B$ appropriately fell as the difference in the probability of the cause prior to $A X$ and $B X$ compounds was systematically increased (Figure 1). This sensitivity to the absolute correlation of the $A$ and $B$ elements with the target event can also be seen in Figure 2, which plots ratings of elements $A$ and $B$ as a function of $p(\mathrm{E} \mid \mathrm{C})$ in Group CE and $p(\mathrm{C} \mid \mathrm{E})$ in Group EC. Although the probability-rating function was a bit less steep in Group EC than in Group CE, there can be little doubt that subjects in each group sensitively discriminated the correlations of elements $A$ and $B$ with the target events; the probability-rating correlation was .997 in Group $C E$ and .981 in Group EC ( $p<.001$ in each case).

Just how are we to understand this difference in subjects' ratings of element $X$ in Groups $C E$ and EC? Wald-

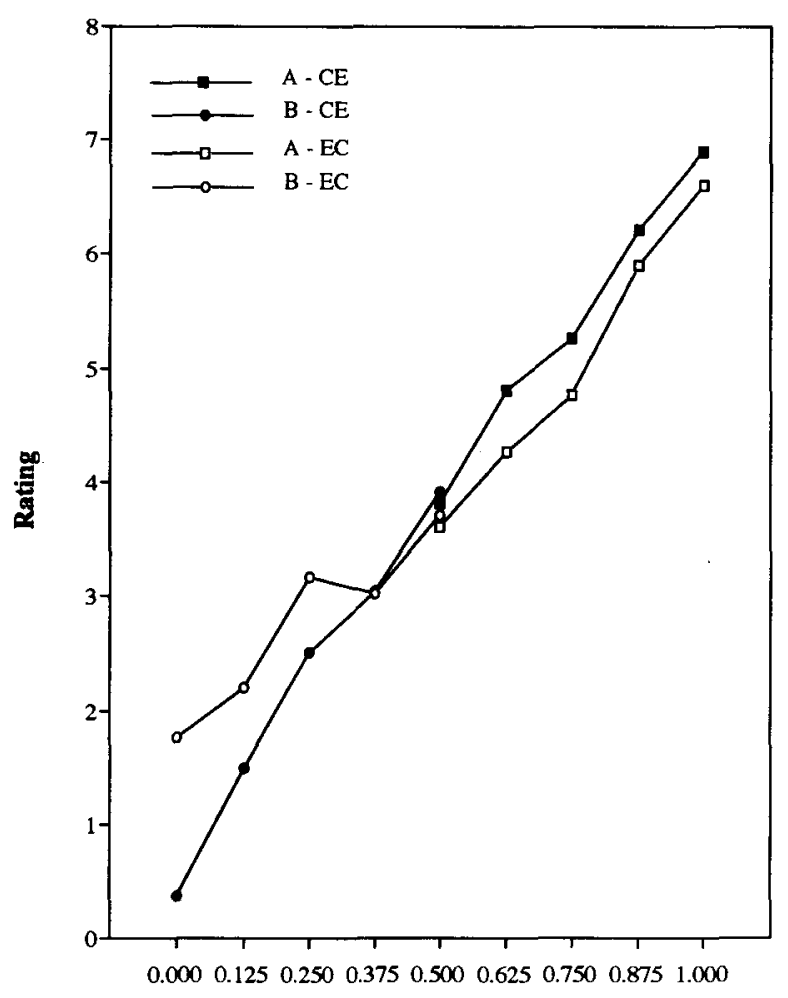

Interevent Correlation

Figure 2. Mean rating scores to elements $A$ and $B$ of $A X$ and $B X$ compounds as a function of $p(E \mid C)$ in Group $C E$ or $p(C \mid E)$ in Group EC. 
mann and Holyoak (1992) propose that different psychological processes mediate subjects' correlational judgments when they are evaluating predictive relations (reasoning from cause to effect) than when they are evaluating diagnostic relations (reasoning from effect to cause). Waldmann and Holyoak note that in the usual diagnostic causal inference experiment, the order of observation of the cause and effect cues is reversed from the actual necessary temporal ordering. Causes must naturally precede effects, but in diagnostic judgment experiments subjects usually "predict" causes from observed effects. Waldmann and Holyoak have further argued that people learn directed links from causes to their effects (rather than from effects to their causes) even in situations in which effect information is received prior to cause information. They assert, however, that the predictions of associative models must be based on the order of observation, since a CS always precedes a US. Therefore, according to Waldmann and Holyoak, in a diagnostic judgment task the CSs are represented by the effects and the US is represented by the potential cause. And, since associative models predict competition between CSs, cue competition should occur between effect cues in these tasks if associative accounts are accurate.

However, our present results (in which cause and effect information was simultaneously available to subjects on a printed page) and those of Waldmann and Holyoak indicate that causal cues compete for associative strength and effect cues do not. Waldmann and Holyoak thus assert that diagnostic causal judgments represent an abstract form of inference which cannot be explained by associative mechanisms. Specifically, they argue that a mentalistic causal-model theory is necessary to explain the fundamental difference between the impact of redundancy for causes and effects: causes compete with one another, but effects do not (and may even collaborate with one another). This disparity is said to arise because people call on preexisting abstract world knowledge in diagnostic judgment tasks.

It is certainly accurate to say that the experimental dissociation of the logical and the observed sequence of events is possible only because of the feasibility of presenting abstract, verbal information to human subjects. But, in our opinion, the capability of humans to convert the order of presented information to the necessary temporal sequence of a causal relation (Waldmann and Holyoak's directed link) does not necessarily preclude the possibility that associative learning is involved.

Waldmann and Holyoak do not tell us precisely how abstract knowledge about causal relations is acquired, nor do they say exactly how this abstract knowledge interacts with concrete situational information to yield subjects' actual judgments (cf. Shanks, 1991b). They do propose that diagnostic inferences (which go from observed effects to possible causes) require cognitive mechanisms that do more than assess cause-effect contingencies. Diagnostic reasoning, they say, requires adjudication among competing causal theories in addition to knowledge of cause-effect contingencies.
These claims are based partly on the results of their own experiments showing that, if only one possible cause of multiple observed effects is available, then cue competition among the effects does not occur. (What Waldmann and Holyoak refer to as "competing causal theories" are actually potential alternative causes for one of the effects, which are said to be derived from prior knowledge.) Waldmann and Holyoak have also shown that when alternative causes are viable as explanations for one of the effects, cue competition among the effects does occur.

Our own results are consistent with those of Waldmann and Holyoak. In Group EC of our experiment, subjects were asked about the effects of one particular food-only one cause was viable-and effect cues did not compete with one another. The results of Shanks (1991a, Experiment 3)-in which effect cues did compete for associative strength-might also be explained within Waldmann and Holyoak's framework by proposing that, in Shanks's study, there may have been viable alternative causes for the relevant effects. The number and type of trials in Shanks's experiment can be represented schematically as follows: Situation $1=\mathrm{AB} \rightarrow$ Disease $1(20), \mathrm{AC} \rightarrow$ no disease (20); Situation $2=\mathrm{DE} \rightarrow$ Disease $2(10), \mathrm{DE} \rightarrow$ no disease (10), DF $\rightarrow$ Disease $2(10), D F \rightarrow$ no disease $(10)$. The 80 trials were presented randomly in a within-subject design. Diseases 1 and 2 were designated in a counterbalanced fashion as Phipp's syndrome and Marshall-Isaacs disease; A, B, C, D, E, and F were various symptoms (e.g., headache or bloody nose). The crucial result was the relative rating of the association between Symptom A and Disease 1 versus the rating of the association between Symptom D and Disease 2. The statistical relations were the same in both cases; the situations differed only in terms of the relative predictiveness of the symptoms paired with A and D. But, as Shanks predicted, subjects rated the relation between $D$ and Disease 2 significantly higher than they rated the relation between A and Disease 1, demonstrating cue competition between effects.

Thus, even though Shanks's design was conceptually quite similar to that used in our Group EC, the results were quite different. We believe that these divergent results can be explained, however, by considering the nature of the questions Shanks's subjects answered at the end of all 80 trials: "If you were to see 100 patients, each of whom had [symptom], then how many of them would you estimate would be suffering from: Phipp's syndrome? MarshallIsaacs disease? No illness?' Even though Symptoms A and $\mathrm{D}$ had been paired with only one of the diseases (and with no illness), subjects were now asked about both diseases as a potential cause ${ }^{1}$ of the symptoms. In addition, specifically asking for ratings of the association of the symptoms with "no illness" may have implied a nonillness cause of the symptoms. In contrast, our Group EC subjects rated only the probability that each symptom was the effect of eating one particular food (shrimp).

Although Shanks's results might, therefore, be viewed as consistent with Waldmann and Holyoak's theory, further research involving multiple explicit potential causes 
with multiple effects will clearly be necessary to completely test Waldmann and Holyoak's assertions about when cue competition among effects will occur.

Certainly, Waldmann and Holyoak have pointed out an important and interesting asymmetry between subjects' predictive and diagnostic inferences: multiple possible causes of a single effect compete with one another, whereas multiple possible effects of a single cause do not. Nevertheless, we see nothing in the mounting evidence for a competitive asymmetry between causes and effects which calls into question an associative explanation of correlational judgments or requires that more complex mechanisms mediate diagnostic as opposed to predictive reasoning. This asymmetry is not exclusive to "higher order" learning tasks or to humans. In conditioning experiments with rats, Rescorla (1991) has demonstrated that multiple outcomes can be associated, without competition or interference, with a single instrumental response.

Both humans and nonhumans undoubtedly observe relations among events in the environment and use some of these events to predict and diagnose others. Nonhumans cannot readily communicate their diagnosis of the cause of an outcome (reinforcement) on a particular individual trial; but, across trials, the strengthening of a response indicates that, in some sense, they have diagnosed that the response "caused" the outcome on prior trials.

It is not surprising that competitive asymmetry between causes and effects is observed in both humans and nonhumans, as this phenomenon can readily be interpreted as due to an adaptive biological mechanism. If one reliable predictor of a biologically significant event (e.g., food or predator) is identified, then continued attention to redundant and less reliable predictors would be inefficient and possibly dangerous. Evolutionary selection for such unselective attention would seem highly unlikely. On the other hand, if one event is the most reliable predictor of two or more subsequent events, then it would not be adaptive for these "effects" to compete with one another; a more adaptive response for the organism would be to associate each effect with its own most reliable predictor. Such evolutionary considerations argue against the dismissal of associative accounts of causal induction.

A final matter merits discussion. The present research involved interevent relations which were presented to subjects in summary form. Individual meal data were shown to subjects as a row-by-row listing, which provided temporal and sequential information without actually giving subjects the findings in a bit-by-bit fashion. There has been rather good empirical convergence in the predictive judgments of individuals given interevent relations in summary form or iteratively (Shanks, 1991b), although more investigation into the situational generality of diagnostic judgments is warranted.

Shanks's (1991b) has argued, however, that this empirical convergence does not necessarily demonstrate that similar mechanisms mediate causal judgments when the information is described in summary form or is accumulated sequentially through experience. Specifically,
Shanks has proposed that associative mechanisms can account only for causal judgments about information that is presented and experienced over time. Abstract causal "metabeliefs" are said to account for judgments of summarized information. Both Shanks's abstract metabeliefs and Waldmann and Holyoak's abstract causal-model theory suggest that the use of an abstract rule precludes the interpretation of some types of causality judgments (those requiring abstract inferences) within an associative framework.

Proponents of connectionist models would, however, describe these rules as emergent regularities (Smolensky, 1988) which develop through interactions with and feedback from the environment over time. And these regularities (e.g., rules, beliefs) emerge through acquisition of the appropriate connection strengths via associative learning processes. According to connectionist theory (and in contrast to some cognitivist explanations), abstract rules are not explicitly stored, retrieved, and used in inference processes; they exist in an implicit manner as emergent properties of a dynamic associative process.

Shanks (1991b) describes causal metabeliefs as involving, in part, abstract world knowledge about the basic characteristics governing causal relations (e.g., temporal precedence, contiguity, necessity [diagnosticity], and sufficiency [predictiveness]). The judgment and problemsolving activities involved in causal inferences about described situations obviously require the abilities of humans to acquire and manipulate verbal and symbolic information. However, Shanks's proposal that causal judgments about described situations cannot result from an associative process seems to be based on more than the necessity of presenting such information in a verbal format. The further assumption is made that the abstraction of rules and beliefs from temporally experienced events cannot result from an associative mechanism.

This further assumption can be contradicted, however, by noting that the causal rules such as contiguity, temporal precedence, necessity, and sufficiency, described by Shanks (1991b) as nonassociative metabeliefs, also represent the conditions necessary for classical and instrumental learning by animals. The temporal precedence of the conditioned stimulus and its contiguity with the unconditioned stimulus are hallmarks of the procedures necessary for classical conditioning to occur. And stimulus selection effects such as blocking, overshadowing, and latent inhibition, in both classical and instrumental conditioning, represent evident conditioning failures due to failures of necessity or sufficiency. Few would argue that these results are due to nonassociative processes or that they require the conscious application of causal rules.

The abstraction of causal rules or metabeliefs through experience and the utilization of such rules in concrete situations are each quite consistent with the connectionist framework, which proposes that complex knowledge (both concrete and abstract) emerges through processes that are ultimately associationistic. Associative and cognitive accounts of human judgment may not be mutually exclu- 
sive competitors; they may merely represent different levels of explanation.

\section{REFERENCES}

Chapman, G. B., \& Robbins, S. J. (1990). Cue interaction in human contingency judgment. Memory \& Cognition, 18, 537-545.

Fales, E., \& WASSERMAN, E. A. (1992). Causation, association, and behavior. Journal of Mind \& Behavior, 13, 1-28.

GLUCK, M. A., \& BowER, G. H. (1988). From conditioning to category learning: An adaptive network model. Journal of Experimental Psychology: General, 117, 227-247.

Hull, C. L. (1943). Principles of behavior. New York: AppletonCentury-Crofts.

ResCoRla, R. A. (1991). Associations of multiple outcomes with an instrumental response. Journal of Experimental Psychology: Animal Behavior Processes, 17, 465-474.

Rescorla, R. A., \& Wagner, A. R. (1972). A theory of Pavlovian conditioning: Variations in the effectiveness of reinforcement and nonreinforcement. In A. H. Black \& W. F. Prokasy (Eds.), Classical conditioning: II. Current research and theory. New York: AppletonCentury-Crofts

Rumelhart, D. E., \& McClelland, J. L. (1986). Parallel distributed processing: Explorations in the microstructure of cognition. Cambridge, MA: MIT Press.

SHANKS, D. R. (1991a). Categorization by a connectionist network. Journal of Experimental Psychology: Learning, Memory, \& Cognition, 17, 433-443.

SHaNKs, D. R. (1991b). On similarities between causal judgments in experienced and described situations. Psychological Science, 2. 341-350.

SHANKS, D. R., \& DiCKINSON, A. (1987). Associative accounts of causality judgments. In G. H. Bower (Ed.), The psychology of learning and motivation (pp. 229-261). New York: Academic Press.
SMOLENSKy, P. (1988). On the proper treatment of connectionism. Behavioral \& Brain Sciences, 11, 1-74.

SPENCE, K. W. (1956). Behavior theory and conditioning. New Haven, CT: Yale University Press.

Wagner, A. R., Logan, F. A., Haberlandt, K., \& Price, T. (1968) Stimulus selection in animal discrimination learning. Journal of Experimental Psychology, 76, 171-180.

W AldMANN, M. R., \& HolyoAK, K. J. (1990). Can causal induction be reduced to associative learning? In Procedings of the Twelfth Annual Conference of the Cognitive Science Society (pp. 190-197). Hillsdale, NJ: Erlbaum.

Waldmann, M. R., \& Holyoak, K. J. (1992). Predictive and diagnostic learning within causal models: Asymmetries in cue competition. Journal of Experimental Psychology: General, 121, 222-236.

Wasserman, E. A. (1974). Stimulus-reinforcer predictiveness and selective discrimination learning in pigeons. Journal of Experimental Psychology, 103, 284-297.

WASSERMAN, E. A. (1990a). Attribution of causality to common and distinctive elements of compound stimuli. Psychological Science, 1, 298-302.

Wasserman, E. A. (1990b). Detecting response-outcome relations: Toward an understanding of the causal texture of the environment. In G. H. Bower (Ed.), The psychology of learning and motivation (pp. 27-82). New York: Academic Press.

\section{NOTE}

1. Shanks's experiment did not explicitly concern causal judgments. Subjects were asked only to estimate the degree of association between particular symptoms and particular diseases. It seems reasonable to assert, however, that a medical diagnostic task at least implicitly involves the assessment of a cause-effect relation.

(Manuscript received May 6, 1992; revision accepted for publication April 16, 1993.) 